\title{
Sex and Pain Perception and Analgesia
}

\author{
Cláudia Carneiro de Araújo Palmeira ${ }^{1}$, Hazem Adel Ashmawi, TSA ${ }^{2}$, Irimar de Paula Posso, TSA ${ }^{3}$
}

\begin{abstract}
Summary: Palmeira CCA, Ashmawi HA, Posso IP - Sex and Pain Perception and Analgesia.
Sex is an important factor in painful experience modulation. Large volume of evidence shows that experience is different for males and females, as well as the answer to some classes of analgesics. Laboratory experiments suggest that women have a lower pain threshold than men related to pain from noxious stimuli such as heat, cold, pressure and electrical stimulation. Pain is a dynamic phenomenon under the influence of various mechanisms of excitatory and inhibitory control. The differences in pain perception related to sex may be associated with hyperalgesia in women, but also to the hypoactivity of the inhibitory system of pain in females. The purpose of this review besides showing some relationship for gonadal hormones, central nervous system and pain is to provide reference points for the discussion of one of the most intriguing aspects of the pathophysiology of pain: the differences in the presence of painful stimuli related to gender.
\end{abstract}

Keywords: Sex Factors; Pain; Gonadal Steroid Hormones.

○2011 Elsevier Editora Ltda. All rights reserved.

\section{INTRODUCTION}

Differences between men and women regarding pain prevalence, prevalence of seeking medical treatment due to pain conditions, as well as pain behavior and response to treatments have long been noted. The role of social, cultural and biological factors concerning the difference in pain perception between the sexes has been discussed. During the last 20 years, a large body of data has been collected concerning differences between the sexes in response to pain, including pain thresholds, and tolerance and response to pain treatments ${ }^{1}$. However, the exact differences as well as their relevance are far from clear.

Sex differences in pain perception have been clearly documented in the literature. It has been shown that women perceived more pain than men ${ }^{2}$. This has been demonstrated regarding clinical pain ${ }^{3}$, experimental pain in humans ${ }^{4}$ and in animal models ${ }^{5}$.

According to the International Association for the Study of Pain, pain is an unpleasant sensory and emotional experience arising from actual or potential tissue damage or described in terms of such damage ${ }^{6}$. This definition does not differentiate

Received from Laboratório de Investigação Médica, LIM-08, Anestesiologia Experimental at Faculdade de Medicina da Universidade de São Paulo (FMUSP), Brazil.

1. Anesthesiologist; Ph.D. student, Faculdade de Medicina da Universidade de São Paulo (FMUSP)

2. Ph.D; Professor Collaborator in Surgery Department, Faculdade de Medicina da USP; Anesthesiologist for the Pain Control Group, Anesthesia Division, Instituto Central do Hospital das Clínicas of the FMUSP

3. Associate Professor, Surgery Department, FMUSP; Supervisor Pain Control Group,

Anesthesia Division, Instituto Central do Hospital das Clínicas of the FMUSP

Submitted on November 16, 2010

Approved on February 21, 2011.

Correspondence to:

Dr. Hazem Adel Ashmawi

Av. Dr. Arnaldo, 455, 20 andar

01246903 - São Paulo, SP, Brasil

E-mail: hazem@hcnet.usp.br between pain as a woman experiences it from pain as a man experiences it, and thus fundamental questions still remain ${ }^{2}$.

Sex differences in pain perception are frequently substantial in magnitude, with moderate to large effect sizes. Our knowledge of sex differences in central nervous system (CNS) morphology and function is becoming more detailed day by day, in both humans and animals 7,8 . Many factors are considered responsible for sex differences in pain perception and for the great prevalence of chronic pain conditions in women 9 ; biological factors such as sex hormones are thought to be one of the main mechanisms which explain sex differences in pain perception. This is supported by numerous findings from the animal ${ }^{10-13}$ and human literature ${ }^{14,15}$.

\section{Epidemiology of sex differences in pain}

Compared with men of similar age, women have a higher risk for stress related-disorders, such as fibromyalgia ${ }^{16}$ and chronic pain ${ }^{17}$. Pain is a frequent symptom of conditions, such as the irritable bowel syndrome, chronic pelvic pain and interstitial cystitis, and is often present without a demonstrable pathology 18,19 . These conditions are highly prevalent in women, with a general occurrence rate in this population of around $3.8 \% 20,21$.

Females report more severe pain, more frequent-bouts of pain, more anatomically diffuse and long-lasting pain than males with similar disease processes, even when male and female specific disorders including male urologic and female gynecologic pain are excluded from the analyses ${ }^{1}$.

Women have a more frequent prevalence of pain related to musculoskeletal or visceral origin, as well as pain related to autoimmune disease. They are more likely to visit a physician and to report pain as a symptom than males ${ }^{22}$, use pain-relieving medications significantly more often even when equating the sexes on pain frequency and severity ${ }^{23}$. 


\section{Physiology of gonadal steroid hormones}

The gonadal steroid hormones are produced by the ovaries and testes (gonads). The main products of the testes are androgens, testosterone, and dihydrotestosterone; and the main products of the ovaries are two types of steroid hormones: estrogens (e.g., estradiol, estriol, estrone) and progestins (e.g., progesterone). Androgens are also produced by the adrenal cortex and are released in response to adrenocorticotropic hormone. Testosterone is a precursor to estradiol, so the ovaries also synthesize testosterone, and the testes also produce some estrogens because estradiol is a metabolite of testosterone.

The aromatization of testosterone to estradiol is greatly facilitated by the enzyme aromatase. This means that tissues containing aromatase can convert testosterone to estrogen and thereby make use of estrogen via estrogen receptors. In women, testoterone is produced in the adrenal cortex $(25 \%)$ and ovaries $(25 \%)$ and by transformation $(50 \%)$ in the liver, kidneys, bowel, lungs, adipose tissue, and CNS ${ }^{24}$.

Menopause induces changes in the endogenous hormone balance: ovarian production of estrogens dramatically decreases. Thereafter, the adrenal cortex becomes responsible for estrogen production via aromatization of androgens to estradiol in peripheral tissue (e.g., fat).

Of functional significance is the fact that many of the actions of gonadal steroids are tissue-specific due to tissue-specific coregulators, proteins that can initiate, amplify or extinguish a transcriptional signal ${ }^{25}$. In addition, steroid hormone receptors may be activated by a variety of factors other than their cognate ligands. Specifically, progestins, androgens and glucocorticoids can bind to each other's receptors. Moreover, gonadal steroid hormone receptors account for a considerable amount of signal diversity because progesterone and estrogen receptors exist in different isoforms ${ }^{26,27}$. Interestingly, estrogens stimulate their own receptor early in development, inhibit it during adulthood, and stimulate it again in the context of brain injury ${ }^{28}$.

\section{Gonadal steroids hormones - CNS, sex differences and pain}

The precise roles of gonadal steroid hormones in pain are not well understood. Many hormone effects on pain/analgesia that were initially thought to be due to gonadal release of hormone may actually be due to de novo hormone synthesis in tissues such as the brain and spinal cord (e.g., progesterone ${ }^{29,30}$, estradiol ${ }^{31-33}$ ). Many of the central regions implicated in pain and analgesia (i.e., periaquedutal gray, spinal cord, dorsal root ganglion) contain receptors for both estrogens and androgens and have the ability to synthesize steroides locally ${ }^{34-36}$. Binding of specific steroids to their receptors within these central and peripheral regions is likely involved in ligand-dependent transcriptional events, thereby influencing the expression of various neurotransmitters and receptors ${ }^{2}$.
Converging line of evidence suggested that sex steroids have profound effects not only on developmental organization but also on ongoing dynamics of the nervous system. In adults, sex steroids affect brain functions by modulating neurotransmission ${ }^{37-39}$. In this case, sex hormones act as neuroactive steroids - this term has been used for steroid hormones that modulate neuronal excitability via specific interactions with neurotransmitter receptors and ion channels ${ }^{40-42}$. The action of neuroactive steroids can be genomic or non genomic. In their genomic action, steroids influence the electrical activity of neurons by regulating the transcription of ion channels and neurotransmitter receptors. Genomic actions require a period longer than $\mathbf{3 0} \mathrm{min}$ to be effective (typically from hours to days) ${ }^{5}$.

In the mid-1970s the observation of rapid and reversible changes in neuronal excitability (in vitro) after application of sex steroids indicated that these hormones could also act via membrane-bound receptors ${ }^{43}$. Because of the rapidity of these non-genomic effects (from milliseconds to minutes), these mechanisms can be studied more easily through electrophysiological techniques ${ }^{38}$. Non-genomic actions include binding of steroids to membrane receptors coupled to ion channels and transmitter receptors by second messengers such as G-proteins, cyclic nucleotides, and kinases or direct allosteric interaction of steroids with neurotransmitter receptors ${ }^{41}$.

However, neuroactive steroids such as estrogens and androgens can be synthesized in the central nervous system de novo from endogenous cholesterol, especially in the hippocampus 44,45 . The term neurosteroid has been coined to define all steroids that are synthesized in the brain ${ }^{46}$. Under this general definition, estradiol would be classified as a neurosteroid since it can be synthesized from circulating testosterone in various brain regions ${ }^{47}$. In contrast to the firstgeneration neuromessengers (such as glutamate, GABA, and acetylcholine) which are stored in presynaptic vesicles and quickly released, neurosteroids are produced in mitochondria and microsomes of neurons and glial cells and are released slowly by passive diffusion. Since neurosteroids also reach cells near the steroidogenic cells, it has been suggested that they can act both as intracrine and paracrine modulators ${ }^{45}$.

In addition to playing a role in activation of the molecular cascade involved in plastic adjustment of cellular functions, estrogens show close interactions, cross-coupling, and reciprocal regulation with proteins involved in signal transduction mediated by neural growth factors or neurotransmitters. For instance, estrogens increase nerve growth factor (NGF) in the dorsal root ganglia ${ }^{48}$, induce $c$-fos expression in the hippocampus ${ }^{49}$, and activate MAP-kinase (a growth factor) not related to estrogen receptors activation ${ }^{50}$.

Estradiol potentiates glutamate binding to N-methyl-D-aspartate (NMDA) receptor ${ }^{51}$ and increases postsynaptic potentials in the hippocampus by increasing currents mediated by kainate receptors ${ }^{52}$; however, inhibitory actions in this structure have also been described ${ }^{38}$.

An interaction of estradiol that is important in the modulation of pain mechanisms is that with the GABA system in the 
CNS, although the effects of estrogens on GABA system are controversial ${ }^{49}$. Changes in estrogen plasma levels are also accompanied by changes in a variety of other neurotransmitters, including serotonin (5-HT), acetylcholine (ACh), dopamine (DA), and $\beta$-endorphins. Thus, the decrease of central 5-HT accompanying estrogen withdrawal may be associated with a reduced self-inhibitory effect at the 5 -HT receptor level. This effect seems to be associated with an increased occurrence of headache ${ }^{53}$.

Regarding androgens, some data suggest reciprocal interactions between androgens receptors and estrogens receptors in the brain, and androgens regulate the activity of the enzyme aromatase in several brain areas ${ }^{54}$. The effects of androgens on neuronal excitability are controversial, and the observed excitatory effects of testosterone are probably due to its conversion to estradiol ${ }^{44}$.

\section{Animal studies}

Animal studies have produced contradictory results regarding the effects of gonadal hormones on pain sensitivity.

In an experiment of ureteral calculosis, a model of visceral pain, female had a higher number and duration of stoneinduced crisis than male, and a treatment with estradiol or testosterone differently affected the behavioral and hormonal parameters in both sexes, supraphysiological levels of estradiol, but not of testosterone, were analgesic only in female 55 . On the other hand, the same group showed that estradiol and androgen receptor antagonists induced analgesia, but only in female ${ }^{56}$.

Surgical ovariectomy (OVX) induces a permanent reduction in estrogen levels generating a hyperalgesic state of slow onset and long duration that can be prevented or reversed by exogenous administration of estrogen, these results were obtained in somatic pain models ${ }^{57}$. Thermal hypersensitivity following OVX in mice is progressive remaining significant for the duration of the experiment (week 10 after surgery), and was also sensitive to estrogen treatment. Interestingly, no difference occurred in mechanical hyperalgesia ${ }^{58,59}$.

Estrogen seems to present a central effect, sex-related differences in glutamate-evoked afferent discharge result from estrogen-related modulation of the activity and/or expression of peripheral $\mathrm{N}$-methyl-D-aspartate (NMDA) receptors in female rats. Estrogen levels in normally cycling rats were positively correlated with NMDA-evoked masseter afferent discharge, and the treatment of OVX female rats with high dose of estrogen significantly increased NMDA discharge. There was a significant sex-related difference in the response of a masseter muscle afferent fibers to activation of peripheral NMDA receptor that was strongly correlated with plasma estrogen levels ${ }^{60}$.

In a model of visceral pain, colorectal nociceptive response was attenuated by intrathecal NMDA antagonist in OVX rats compared to OVX with estradiol replacement rats. It was concluded that estradiol increases spinal processing of visceral nociception by increasing NMDA receptor NR1 subunit ex- pression and increasing site specific receptor phosphorylation in the NR1 subunit contributing to an increase in NMDA receptor activity 61 .

In a model of painful peripheral neuropathy induced by ethanol in rats, ethanol induced hyperalgesia with more rapid onset and severity in females. Following OVX, alcohol failed to induce hyperalgesia, however, estrogen replacement reinstated alcohol neuropathy in the female rat 62 .

Formalin pain model, a nociceptive and inflammatory pain model, has been widely used for the study of sex differences in pain perception. In a study of hormone replacement in gonadectomized (GDX) female and male rats to observe the effects of sex hormones on excitatory and inhibitory phases of formalin-induced nociceptive responses, the nociceptive responses were divided into three distinct phases: acute (phase I), inhibitory (interphase), and tonic (phase II). It was observed that testosterone had a hypoalgesic effect on phases I and II of the formalin test, female hormones acted only on the interphase: The results permitted to believe that testosterone plays a protective role in pain perception ${ }^{11}$. Female gonadal hormones regarding the responses to repetition of a nociceptive stimulus in the formalin test showed that these hormones affect behavioral and neuronal responses to repeated nociceptive stimulation, indicating a possible role of ovarian hormones in determining sex differences in pain 63 .

In another experiment on formalin-induced behavior during phase I and II, the effects of graded doses of estradiol or progesterone were examined in OVX rats. Graded doses of estradiol reduced formalin-induced behavior by $35 \%$ to $49 \%$ during phase II but not during phase I. The maximal response was seen with $20 \%$ estradiol. The antihyperalgesic effect of $20 \%$ estradiol was significant at eight days after implantation and persisted after 21 days. In contrast, graded doses of progesterone had no effect on either phase of formalin pain induced behaviors. Tamoxifen, an estrogen receptor antagonist, completely prevented the antihyperalgesic effect of the $20 \%$ estradiol implant. Formalin-induced behavior during phase II were significantly less in proestrus females and OVX rats given $20 \%$ estradiol compared to OVX control rats 64 . Focusing on the effects of the estrous cycle in middle-aged (11 months old) rats with unchanged serum estradiol levels in the response to formalin, and analyzing c-Fos expression in order to determine the cerebral region associated with middle-age-related changes in pain response, it was found that middle-aged female rats are more sensitive to formalin-induced nociceptive stimuli, irrespective of estrous cyclicity ${ }^{13}$. They speculate that the hypersensitivity to nociceptive stimuli is due to age, and this response is unrelated to reproductive ability.

In an experiment investigating whether testosterone mediates the antinociceptive effect of clonidine in the trigeminal region of male rat, the results showed that orchiectomy, and thus the absence of testosterone, resulted in a complete lack of an effect of clonidine treatment in males. Testosterone replacement in GDX rats restored clonidines antinociceptive effect. The abolition of clonidines antinociceptive effect in the absence of testosterone was observed on both NMDAinduced nociceptive scratching behavior and on heat-evoked 
head withdrawal test, suggesting that testosterone is essential for the expression of clonidines antinociceptive effects on different modalities of pain processing 65 .

Hippocampal TRPV1 (transient receptor potential vanilloid1) expression in OVX rats that received estradiol was investigated, and estradiol potentiated hippocampal TRPV1 expression and mechanical allodynia of inflamed TMJ ${ }^{66}$. Their results suggested that estradiol could modulate TMJ pain through the TRPV1 signaling pathway in the hippocampus.

In a study to determine whether hormonal modulation of opioid antinociception in adult rats extends to opioids other than the prototypic mu agonist morphine, gonadectomized (GDX) male and female rats were replaced with estradiol, progesterone, testosterone, and no hormone. Nociception was evaluated on the $50^{\circ} \mathrm{C}$ hot plate and warm water tail withdrawal tests before and after subcutaneous administration of hydromorphone, buprenorphine, U50, 488, or SNC80. In males, gonadectomy tended to decrease, and testosterone tended to increase antinociception produced by 3 of the 4 agonists. In females, gonadectomy and hormone treatment had more variable effects, although estradiol tended to decrease $\mathrm{mu}$ opioid antinociception. These results suggested that reproductive hormones such as testosterone in males and estradiol in females do not consistently modulate sensitivity to the analgesic effects of opioids in the adult organism ${ }^{67}$.

Interestingly, the more potent analgesic responses of morphine and clonidine in male mice failed to be observed in mice with a "knock-out" of G protein-coupled inwardly rectifying potassium channel subtype (GIRK-2), suggesting a role for this molecular modulator in the expression of sex differences ${ }^{68}$.

Investigating systematically whether activation of opioid receptor-like 1 receptor (ORL1) by orphanin FQ (OFQ) produced sex-specific modulation of spinal nociception and whether estrogen or testosterone contributes to these differences in two behavioral models in rats, using the NMDA and heat-induced nociceptive tests; it was found that intrathecal microinjection of OFQ in male, OVX, and diestrous (low estradiol) rats produced a significant antinociceptive effect on both tests. OFQ failed to produce antinociception in proestrous rats, the phase of the estrous cycle with the highest levels of circulating estradiol, and produced a dose-dependent effect in OVX females treated with $1 \mathrm{ng}$ to $100 \mu \mathrm{g}$ of estradiol. OFQ was ineffective in GDX males, whereas testosterone replacement restored the antinociceptive effect of OFQ in GDX males. They concluded that ORQ produces sex-specific modulation of spinal nociception; estrogen attenuates antinociception in female in parallel with normal cycling of estrogen levels, and testosterone is required for the expression of antinociception in male 69 .

In mices of both sexes lacking the estrogen receptor $\alpha$ and $\beta$ in respective wild types, nocipetive responses to mechanical stimulation under normal conditions were assessed after inflammation of a hindpaw or peripheral nerve injury. They observed a sex difference in withdrawal threshold to mechanical stimulation in both sets of wild-type mice, with females exhibiting significantly lower response than males. This difference was seen in both strains of wild-type mice. Carrageenan- induced inflammation of a hindpaw, all wild-type and knouckout mice exhibited similar local edema with no difference between sexes. Hypersensitivity (allodynia) to mechanical stimulation was developed in the wild-type mice, witch was more profound in females than in males. Again, such sex difference was not observed in the knock-outs of either estrogen receptor. Photochemically induced partial sciatic nerve injury caused similar persistent mechanical hypersensitivity in the wild types and both estrogen receptor knock-outs with no difference between the sexes. These results suggested that sex difference in basal mechanical pain threshold and inflammatory hypersensitivity is eliminated in mice lacking either the estrogen $\alpha$ receptors or $\beta$ receptors. However, these receptors do not seem to be directly involved in mediating pain sensitivity in general or in the development of neuropathic pain. It was not clear whether the elimination of sex differences observed in the knock-outs reflects an ongoing effect of estrogen acting through its receptors in females or the developmental changes that predominantly affect females ${ }^{70}$.

\section{Gonadal steroid hormones and opioid analgesia}

The endogenous opioid neurotransmitters and $\mu$ opioid receptors are centrally implicated in responses to stress, in the suppression of pain, and in the action of opiate analgesic drugs ${ }^{71}$. Brain regional mu-opioid receptor concentrations have also been shown to differ between men and women, and may also be regulated by age and circulating gonadal steroids 72 .

Gonadal hormones and endogenous pain control are known to be interrelated. Receptors for opioids and gonadal steroids are colocalized on neurons in the central and peripheral nervous systems, and can modulate each other. Both $\alpha$ and $\beta$ estrogen receptors are found on sensory neurons and in the CNS, and the endogenous opioid system is modulated by estrogen and testosterone ${ }^{73}$.

Gonadal steroid hormones can influence sensitivity to analgesia during development of the organism (organizational effects) and/or during adulthood (activational effects) ${ }^{74,75}$. There are numerous studies reporting that the variability in responses to opioids is attributable to subject-patient sex. Human experiments consistently show that $\mu$ opioids have increased potency in females ${ }^{76}$. Some studies of sex differences in opioid analgesia showed that women given opioids have significantly greater analgesia than men after dental surgery ${ }^{77}$.

The periacqueductal gray (PAG) and its descending projections to the rostral ventral medulla (RVM) and spinal cord dorsal horn constitute an essential neural circuit for opioid based analgesia, but the neural mechanisms underlying the sexually dimorphic actions of morphine remain poorly understood ${ }^{78}$.

Including gender as an independent variable, it is almost clear that morphine does not produce the same degree of antinociception in male and female, especially following the induction of persistent pain ${ }^{79}$. Interestingly, while animal studies show a tendency for opioids to act more efficaciously in male, human studies are less clear in the presence and direction of any sex effect ${ }^{78}$. 


\section{Human studies}

Experimental and clinical data indicate the involvement of sex hormones in many pain syndromes, however, like animal studies, human studies have produced contradictory results regarding the effects of gonadal hormones on pain sensitivity.

Studying the influence of an individual gender role expectations of pain (GREP) on experimental pain report, female and male subjects underwent thermal testing and were asked to report pain threshold, pain tolerance, visual analogue scales (VAS) ratings of pain intensity and unpleasantness, and a computerized VAS rating of pain intensity during the procedure. This study revealed two primary findings. First, significant sex differences emerged for measures of pain threshold, pain tolerance, and pain unpleasantness. Second, after statistically controlling for age GREP scores were significant predictors of threshold, tolerance, and pain unpleasantness. Male indicated significantly greater pain endurance than females, while females reported greater willingness to report pain ${ }^{80}$.

Another study evaluated how men and women differ in their response to interdisciplinary chronic pain management. Chronic pain patients who completed a series of measures relating to pain and distress at three different time points: immediately prior, on completion, and three months following an interdisciplinary pain management intervention were analyzed. The results showed that the pain management intervention produced improvements in a range of domains of outcomes for both men and women, such effects were sustained three months following treatment. However, complete reduction was only maintained at the 3-months follow-up time point in men. Women exhibited a return towards baseline levels on these three measures. This study suggested that gender plays a role in reports of pain and distress following interdisciplinary chronic pain management ${ }^{17}$. Interestingly is that there are inconsistencies in the results. Women, but not men, benefited more from an intervention ${ }^{81,82}$, while in other study male patients with low back pain benefited most from physiotherapy and women benefited from back exercises ${ }^{8}$. Thus, although gender differences in treatment effect for pain and distress were found, more research is needed to determine whether such differences are of actual importance in terms of day-to-day performance.

Epidemiology, comorbidity, and impact on health-related quality of life of self-reported headache and musculoskeletal pain, from a gender perspective, were analyzed in a Swedish study ${ }^{23}$. They studied prevalence of pain conditions, especially headache, and found that it was higher among women who also reported more severe pain. Comorbidity between pain conditions and psychiatric or somatic problems was higher among women. Health-related quality of life (HRQoL) differed by gender and type of pain condition. The physical dimensions of HRQoL were more affected by headache among men; psychological dimensions were more affected among women. In both sexes, pain conditions were associated with poorer socioeconomic conditions and life-style factors but there were gender differences.
Pain may be influenced by the menstrual cycle. While the mechanisms underlying these effects are unclear, it is possible that menstrual phase-related changes contribute to endogenous pain modulation. Studying the response to cold pressor, heat, and ischemic pain test in men and women, each woman was tested at three points in their menstrual cycle, the early follicular, late follicular, and luteal phases. Women were significantly more sensitive to cold pain, heat pain, and ischemic pain than men, however, pain perception during each task was not influenced by the menstrual cycle in women, nor did the menstrual cycle influence the magnitude of the gender differences in pain sensitivity ${ }^{84}$. Another study evaluated the levels of sex hormones and pain sensitivity at different phases of a menstrual cycle in regularly menstruating women with fibromyalgia age-matched to healthy women. They showed that women with fibromyalgia had lower pain threshold and tolerance relative to healthy women throughout the menstrual cycle. The authors suggested that the disproportionate prevalence of females with fibromyalgia is not likely to be attributable to hormonal factors ${ }^{85}$.

Many studies show that women are more sensitive to pain than men $4,86,87$. Of the different types of experimental pain, pressure pain in particular seems to be sensitive to sex differences. In a meta-analysis of gender differences in pain report ${ }^{4}$, pressure pain had one of the highest effect sizes. In an experimental pain study that employed the mechanical pressure test in order to examine potential differences in pain report among men, normally menstruating women, and women taking monophasic oral contraceptives, the results demonstrated subtle menstrual-cycle effects in normally menstruating women and women taking monophasic oral contraceptives. Sex differences were few with more group differences and trends emerging between women taking oral contraceptives and men, as opposed to men and normally menstruating women ${ }^{88}$.

The diversity of the gonadal hormones effects implies that regions of the brain outside the hypothalamus are involved. The hypothalamus has been the traditional site for the study of ovarian steroid receptors and their role in the control of reproductive function ${ }^{52}$. The association between supraphysiological levels of estradiol and response patterns to experimental pain was analyzed via quantitative sensory test (QST) ${ }^{89}$. $A$ repeated measures design was used on 31 women, treated with the same in vitro fertilization protocol. Interestingly, great pain response was associated only with supraphysiological levels of estradiol, no other differences in pain perception between the different hormonal levels were observed.

One factor that has been neglected in many previous studies is the effect of sex hormones on endogenous pain inhibitory mechanisms. Sex differences in pain perception may be due to hyperalgesia in women but could also be related to hypoactivity in women's pain inhibitory system. Diffuse noxious inhibitory control (DNIC), an endogenous pain control mechanism ${ }^{90}$, is of particular interest since a deficit in DNIC is associated with the development of chronic pain ${ }^{91}$. In a study where the aim was to develop a simple experimental design that would enable to elicit and measure multiple nociceptive 
mechanisms reported to play a role in the development and persistency of chronic pain, such as temporal summation (TS) and DNIC, eighty-three healthy subjects of both sexes were examined for pain perception. It was found that the mean pain ratings and peak pain but not TS were significantly reduced by DNIC. No sex differences were observed in DNIC analgesia ${ }^{92}$. Previous studies on sex difference in DNIC have yielded equivocal results: two studies have shown no sex differences in DNIC effectiveness ${ }^{93,94}$, while four studies did show greater DNIC in men ${ }^{95-98}$. The divergence in these results may be explained by the use of different experimental designs and different methods to quantify DNIC effectiveness. Further research is needed in order to better understand endogenous pain inhibitory mechanism in men and women.

Trying to establish whether pain perception and DNIC vary across the menstrual cycle, the study of excitatory and inhibitory pain mechanisms during the menstrual cycle in healthy women, showed that significantly more pain inhibition (DNIC effectiveness) during the ovulatory phase occurs compared to the menstrual and luteal phases ${ }^{9}$. The main finding of their study was the observation that only inhibitory mechanisms (DNIC analgesia) and not excitatory pain mechanisms vary throughout the menstrual cycle. The mean reduction in pain intensity was greater during the ovulatory than the menstrual phase. They concluded that DNIC varies with menstrual cycle.

Recently, clinical studies in humans have also reported sex differences in morphine analgesia. Of the limited number of studies that examined gender or sex as an independent variable, it has been reported that males experience greater morphine analgesia compared to females ${ }^{99,100}$. In fact, one study reported that females required $30 \%$ more morphine to reach the same level of analgesia as male ${ }^{99}$. Sex influences analgesic responses to different drugs. K-receptor agonists appeared to be more potent analgesics in women than in men. After surgical removal of the third molar tooth, the k-opioid agonist pentazocine and other k-opioid agonists had significantly stronger analgesic effects in women than in men ${ }^{101}$. $\mathrm{K}$-opioid agonists had an antianalgesic effect in men, increasing their postoperative pain ${ }^{77,102}$. In another interesting study, women who had two variants of the melanocortin-1 receptor (Mc1r) gene, associated with red hear and fair skin, displayed altered k-opioid analgesia. They found that women with two variant $\mathrm{Mc} 1 \mathrm{r}$ alleles displayed significantly greater analgesia from the k-opioid, pentazocine, than women without variants, while in men, pentazocine produced modest analgesia ${ }^{103}$.

While it is clear that sex differences in opioid analgesia are not a simple and straightforward phenomenon, when sex differences are reported they are not trivial in magnitude ${ }^{104}$.

Imaging studies of the brain have shown differences between men and women in the spatial pattern and intensity of response to acute pain. Brain activity in young women and men in response to a painful stimulus (injection of hypertonic saline into the masseter muscle) and in response to carfentanil, a $\mu$-receptor agonist, presents different patterns. The painful stimulus was injection of hypertonic saline into the masseter muscle, with the volume adjusted so that the response of women to pain was similar to that of men. Women were studied during the follicular phase of the menstrual cycle, when pain sensitivity is lowest, again so that their pain response would be similar to that of men. The results demonstrated that at equivalent levels of pain intensity, men and women differ in the magnitude and direction of response of the mu-opioid system in distinct brain nuclei ${ }^{71}$.

Examining both baseline mu-opioid receptor levels and the activation of this neurotransmitter system during sustained pain using positron emission tomography in a sample of young healthy men and women, women were studied twice, during low and high estrogen states. The high-estrogen state was associated with regional increases in baseline mu-opioid receptor availability in vivo and a greater activation of endogenous opioid neurotransmission during the pain stressor. The latter did not differ from that obtained in males. During the low estrogen conditions, however, significant reductions in endogenous opioid tone were observed at the level of thalamus, nucleus accumbens, and amygdala, which were associated with hyperalgesic responses. Estrogen-associated variations in the activity of mu-opioid neurotransmission correlated with individual ratings of the sensory and affective perceptions of the pain and the subsequent recall of that experience. It was demonstrated a significant role of estrogen in modulating endogenous opioid neurotransmission and associated psychophysical responses to a pain stressor in humans ${ }^{105}$.

\section{CONCLUSIONS}

Pain perception is influenced by complex interactions between biological variables - gonadal hormones, genetics, pain circuitry pathways and CNS variation, and psychosocial variables - depression, anxiety, culture, sex role expectancies, social learning factors, pain related appraisal. There are substantial differences in these variables within individuals, and from the point of gonadal hormones and pain, the variability is profound.

This review is summary of an enormous and growing field. Both the animal and human literatures are rife with disagreement and conflicting findings within species and, more important, among species. More researches need to be done to elucidate the basic biological and psychosocial factors responsible for the differences that are described. These are important questions that should be analyzed not just for the joy of intellectual and scientific curiosity, but also to allow better therapy to our female and male patients. 